\title{
Yet Another Approach to the Gough-Stewart Platform Forward Kinematics
}

\author{
Josep M. Porta and Federico Thomas
}

\begin{abstract}
The forward kinematics of the Gough-Stewart platform, and their simplified versions in which some leg endpoints coalesce, has been typically solved using variable elimination methods. In this paper, we cast doubts on whether this is the easiest way to solve the problem. We will see how the indirect approach in which the length of some extra virtual legs is first computed leads to important simplifications. In particular, we provide a procedure to solve 30 out of 34 possible topologies for a Gough-Stewart platform without variable elimination.
\end{abstract}

\section{INTRODUCTION}

A general Gough-Stewart platform consists of six extensible legs attached to a static base and a moving platform at six arbitrary fixed attachment points through spherical joints [1]. The forward kinematics problem consists of determining the pose (position and orientation) of the moving platform relative to the base given the length of the legs, and the coordinates of the attachment points in their local reference frames. Algebraic geometric methods have shown that this problem has 40 solutions [2], [3], [4], all of which can be real [5]. Since the methods to obtain these 40 solutions are quite involved, several researchers have examined the use of additional sensors to uniquely determine the true pose of the moving platform, and to simplify the solution. One example is the work of Bonev et al. [6], who use three additional distance measurements from passive legs, together with the assumption of a planar moving platform, to derive an expression with a unique solution for the relative pose. More recently, Trawny et al. [7] have shown that four additional distance measurements are enough to obtain a unique solution for a generic platform. Nevertheless, this is so for additional distances between arbitrary points. If the points are carefully chosen, the required set of extra distances reduces to three. Indeed, if we consider a general GoughStewart platform, the additional distances corresponding to the three segments in dashed lines appearing in Fig. 1 permit the determination of the coordinates of points $a, c$, and $e$, which define the pose of the moving platform, by performing three trilaterations [8]. Taking the triangle $\triangle_{b d f}$ as a reference, the following simple procedure is applied:

1) Two possible sets of coordinates for point $a$ can be obtained from the length of the leg connecting points $a$ and $b$, and the additional distances $a d$ and $a f$.

The authors are with the Institut de Robòtica i Informàtica Industrial (IRI), CSIC-UPC, Llorens Artigas 4-6, 08028 Barcelona, Spain \{porta, fthomas\}airi.upc.edu

This work has been partially supported by the Spanish Ministry of Economy and Competitiveness through projects DPI2014-57220-C2-2-P, DPI2017-88282-P, and MDM-2016-0656.

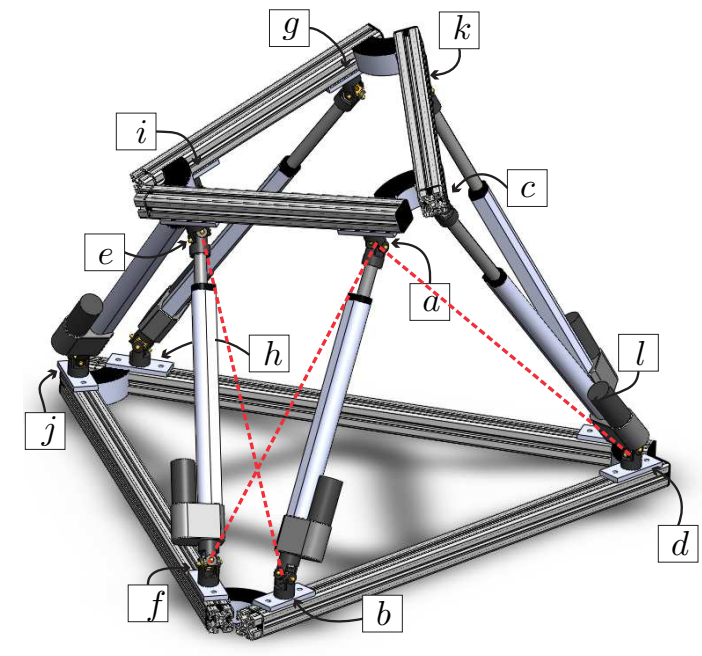

Fig. 1. Gough-Stewart platform whose forward kinematics can be solved by trilateration if the lengths of the segments in dashed line are known.

2) The coordinates of $e$ can be determined, taking $\triangle_{f a b}$ as a reference, and the fixed distances $e f$ and $e a$ and the additional distance $e b$. The two valid solutions in this step applied over the two solutions of the previous step, yield four solutions.

3) Finally, the coordinates of $c$ can be computed, taking $\triangle_{a d e}$ as a reference, and the known distances $c a, c d$, and $c e$. With this, we come up with eight solutions.

This is the situation for the general Gough-Stewart platform. However, it is important to observe that the forward kinematics of some platforms with particular topologies can be solved by trilaterations without requiring any additional distance. These particular platforms are called trilaterable for obvious reasons [9], [10] and, despite their simplicity, they have interesting properties [11].

If we exclude the trilaterable platforms and the general 6-6 case, all others require 1 or 2 additional distances to be solvable by trilateration. In this paper, we discuss this kind of platforms, and we present a procedure for solving the forward kinematics of any member of the class that requires only one additional distance as follows: (1) the additional distance is obtained from all other known distances as roots of a closure polynomial; and (2) the forward kinematics is trivially solved by trilateration. This procedure is more general than the one introduced in [12], in the sense that now we can fix the orientation of arbitrary tetrahedra in the problem. Due to this limitation, the approach presented in [12] 
could only provide minimum degree closure polynomials in particular situations (e.g., when the points in the base and in the platform are coplanar). A closely related procedure to the one described here was recently presented in [13]. Here, a new matrix formulation is introduced leading to important simplifications. Moreover, a novel classification of parallel platform topologies is presented based on classes with a representative, from which all other members of the class can be deduced.

This paper is organized as follows. In the next section, we identify all platform topologies requiring 0,1 or 2 additional distances and we show that the class of platform requiring one distances is composed of two disjoint subclasses with one representative each. Then, in Section III, we describe the procedure to generate a polynomial whose roots correspond to the valid values for the additional distance. In Section IV, we apply this procedure to solve the forward kinematics of the two representative platforms previously identified. Finally, in Section V, the main points presented in this paper are summarized.

\section{Classes of Gough-Stewart Platforms}

It is possible to design simplified versions of the general platform by coalescing some leg end-points. These simplifications are usually identified by the number of attachments in the fixed base and the moving platform. Actually, there are 34 different possible topologies, first identified in [14], which can be classified into the classes 6-6, 6-5, 6-4, 6-3, 5-4, 5-3, 4-3, and 3-3. However, this classification does not permit the identification of the topology of the platform. For example, the following three topologies are possible for a 3-3 platform

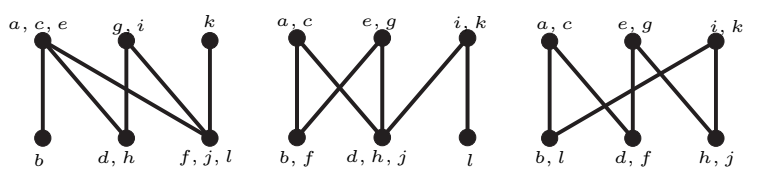

It is possible to divide all possible topologies given in [14] into those requiring $0,1,2$, or 3 additional distances to become trilaterable. In what follows, we will say that these distances correspond to the lengths of virtual legs, in contrast to the real legs lengths defining the problem.

Table II shows all possible topologies for a trilaterable platform. This table also includes a possible sequence of trilaterations as a sequence of tetrahedra. In an abuse of language, the notation $\triangle_{a, b, c, d}$ also denotes a trilateration where the coordinates of point $d$ are obtained from those of points $a, b$, and $c$. Moreover, if one of these tetrahedra is enclosed in brackets, we mean that its orientation is fixed, i.e., we get only one solution for the corresponding trilateration.

Among all the trilaterable topologies, we can select the
TABLE I

ALL POSSIBLE TOPOLOGIES FOR A TRILATERABLE STEWART-GOUGH PLATFORM.

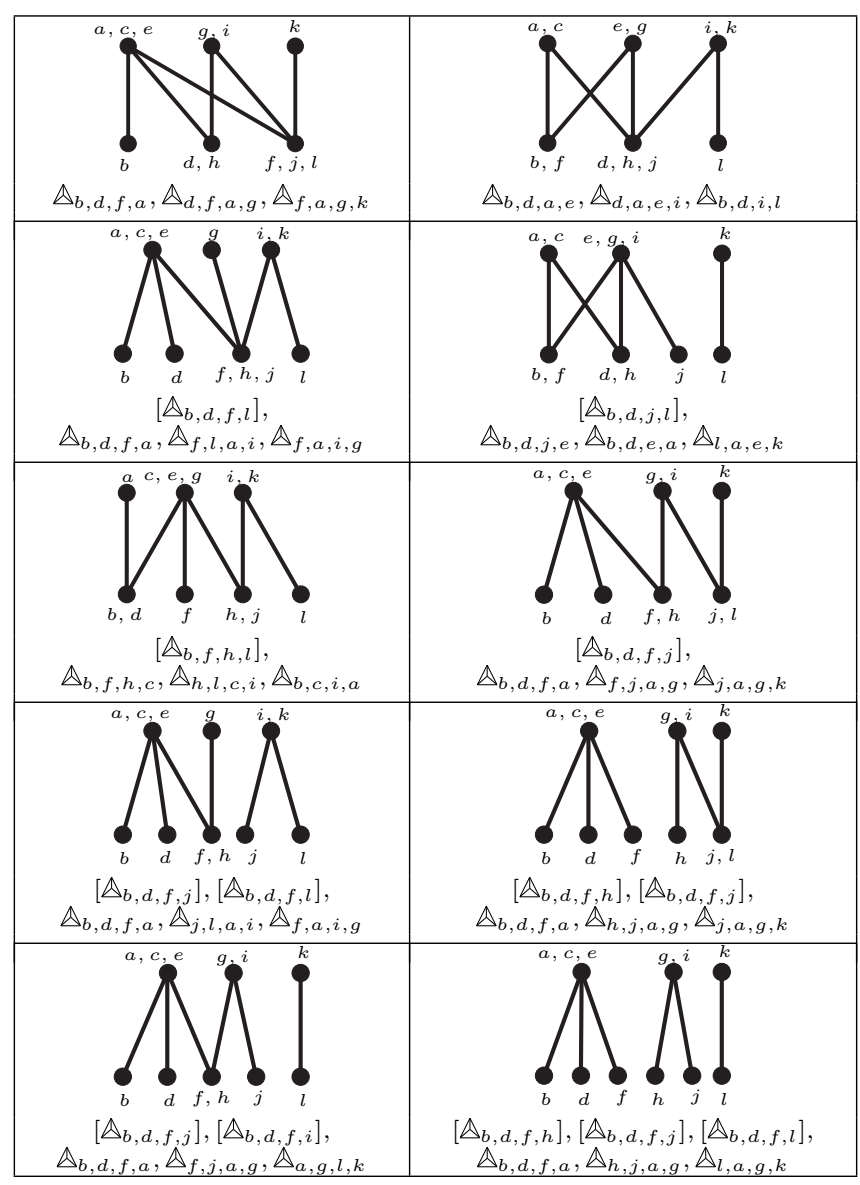

following one as the representative

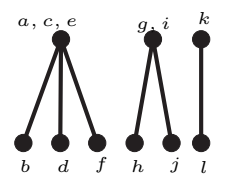

because all other topologies in the class can be seen as simplifications where some attachments merge without leading to a degenerate platform. A general method for solving the forward kinematics of the representative is also applicable to all other member in the class and hence its interest.

Tables II and III show all topologies for platforms whose forward kinematics can be solved via trilateration when properly adding one virtual leg. For each case, one valid virtual leg is shown in dashed line, and a possible trilateration sequence is also indicated. In all the topologies in Table II, three real leg attachments coincide. The length of the three involved legs control the position of a point in the moving platform and the remaining three legs define the platform orientation about this point. In contrast, in the topologies in Table III there is not a direct relation between particular leg lengths and the position/orientation of the platform. Observe that both classes are disjoint. While, the representative of the 
TABLE II

All possible topologies for a Decoupled Stewart-Gough PLATFORM. THEY ONLY REQUIRE ONE VIRTUAL LEG, E.G., THE ONE SHOWN IN DASHED LINE, TO BECOME TRILATERABLE.

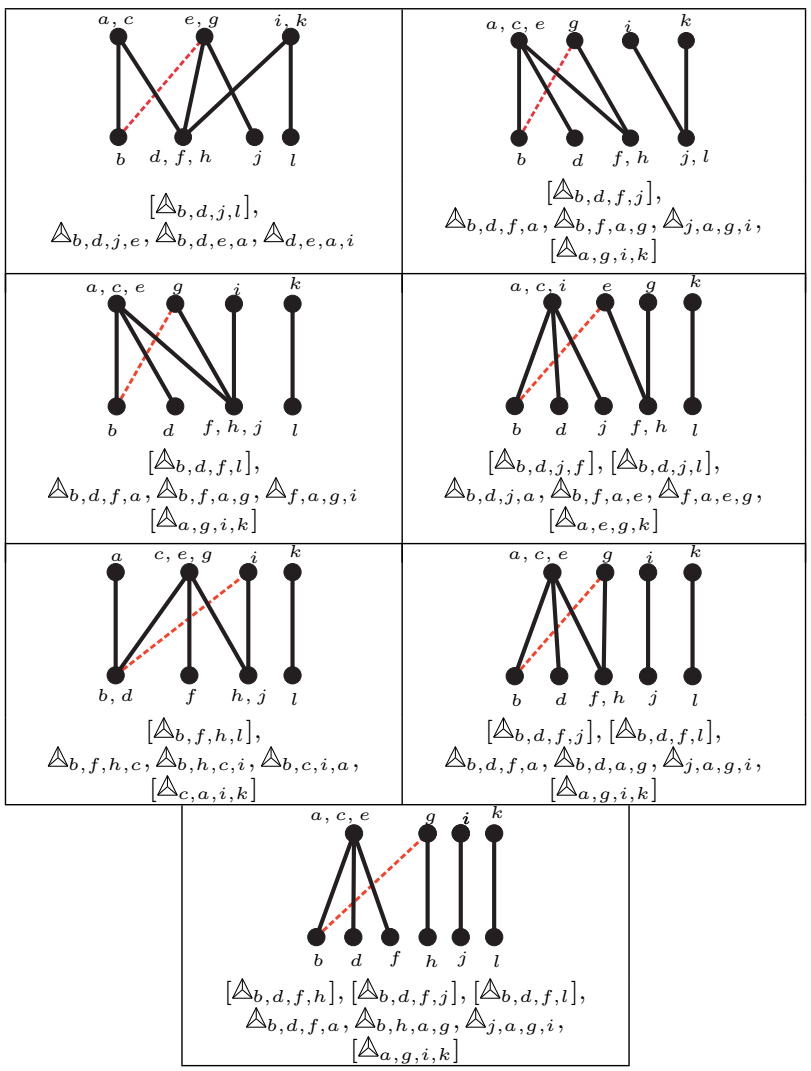

class in Table II is

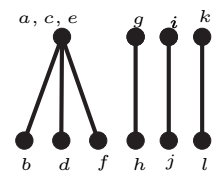

the representation of the class in Table III is

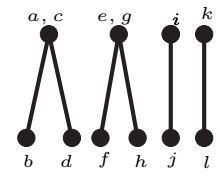

Finally, Table IV includes the platform topologies that need two virtual legs to become trilaterable. The representative for this class is

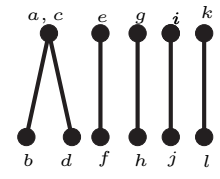

To solve the forward kinematics of the platform using trilateration, first we need to fix the length of the added virtual legs. Since the real leg lengths define a discrete set of valid platform poses, and the platform pose unequivocally

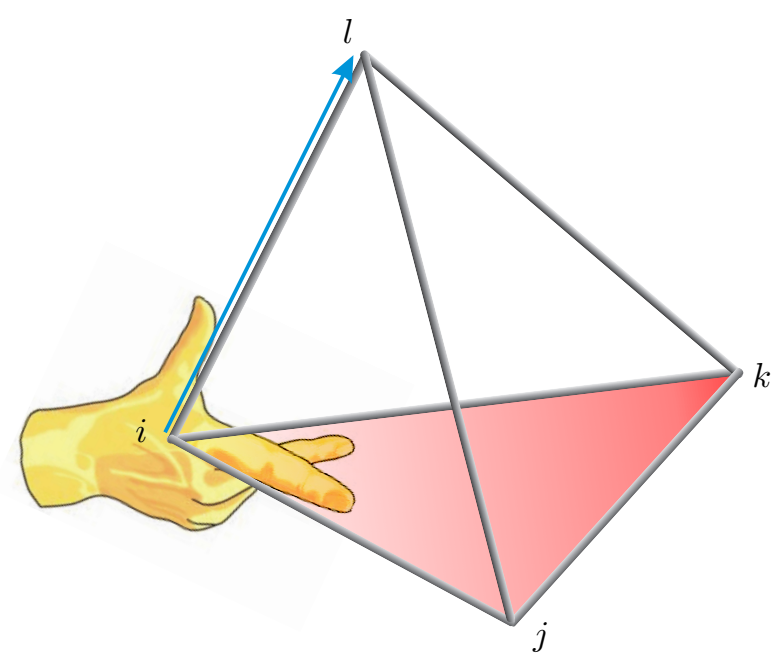

Fig. 2. The trilateration step denoted as $\bigotimes_{i, j, k, l}$, has its origin at $i$, its base is given by then triangle $\triangle_{i, j, k}$, its base vectors are $\mathbf{p}_{i, j}$ and $\mathbf{p}_{i, k}$, and its output vector is $\mathbf{p}_{i, l}$. These vectors are always chosen following the right hand rule $\left|\mathbf{p}_{i, j} \mathbf{p}_{i, k} \mathbf{p}_{i, l}\right|>0$.

defines the virtual leg lengths, arbitrary values of the virtual leg lengths are not valid. Next, we describe how to identify the valid values as the roots of a minimum degree closure polynomial. These roots are fed into the trilateration process to obtain the valid poses of the platform. In this paper, we focus on the platforms requiring only one virtual leg thus covering most parallel platform topologies (20 out of 24 , if trilaterable topologies are excluded).

\section{The Geometry of Strips of Oriented TETRAHEDRA}

In what follows, $P_{i}$ will denote a point in $\mathbb{E}^{3}, \mathbf{p}_{i}$ a column vector with its coordinates in a given reference frame, $\mathbf{p}_{i, j}=$ $\mathbf{p}_{j}-\mathbf{p}_{i}, \mathbf{p}_{i, j, k}=\mathbf{p}_{i, j} \times \mathbf{p}_{i, k}$, and $s_{i, j}=\left\|\mathbf{p}_{i, j}\right\|^{2}$. To light the notation, $P_{i}$ will be also denoted as $i$ when no confusion is possible. Vectors $\mathbf{p}_{i, j}, \mathbf{p}_{i, k}$, and $\mathbf{p}_{i, j, k}$ represent, in general, a non-orthonormal reference frame (Fig. 2), which will be denoted by the matrix

$$
\mathbf{Q}_{i, j, k}=\left[\mathbf{p}_{i, j} \mathbf{p}_{i, k} \mathbf{p}_{i, j, k}\right] .
$$

The trilateration step $\triangle_{i, j, k, l}$ is said to have its origin located at $i$, its base, $\mathbf{Q}_{i, j, k}$, is given by the triangle $\triangle_{i, j, k}$, its base vectors are $\mathbf{p}_{i, j}$ and $\mathbf{p}_{i, k}$, and its output vector is $\mathbf{p}_{i, l}$, which can be used to determine the coordinates of $l$ in $\mathbf{Q}_{i, j, k}$ since $\mathbf{p}_{l}=\mathbf{p}_{i}+\mathbf{p}_{i, l}$.

The Cayley-Menger bi-determinant of two sets of points, $i_{1}, \ldots, i_{n}$ and $j_{1}, \ldots, j_{n}$, is defined as

$$
D\left(i_{1}, \ldots, i_{n} ; j_{1}, \ldots, j_{n}\right)=\left|\begin{array}{cccc}
0 & 1 & \ldots & 1 \\
1 & s_{i_{1}, j_{1}} & \ldots & s_{i_{1}, j_{n}} \\
\vdots & \vdots & \ddots & \vdots \\
1 & s_{i_{n}, j_{1}} & \ldots & s_{i_{n}, j_{n}}
\end{array}\right| .
$$

If the two sets of points are the same, then $D\left(i_{1}, \ldots, i_{n}\right)=$ $D\left(i_{1}, \ldots, i_{n} ; i_{1}, \ldots, i_{n}\right)$ is called the Cayley-Menger determinant of the involved set of points. An alternative common definition of the Cayley-Menger determinants includes 
TABLE III

All possible topologies, that ARE ARE NOt DeCOUPled, FOR A STEWART-Gough PLATFORM WHich REQUiRE ONE VIRTUAL LEG, E.G., THE ONE SHOWN IN DASHED LINE, TO BECOME TRILATERABLE.

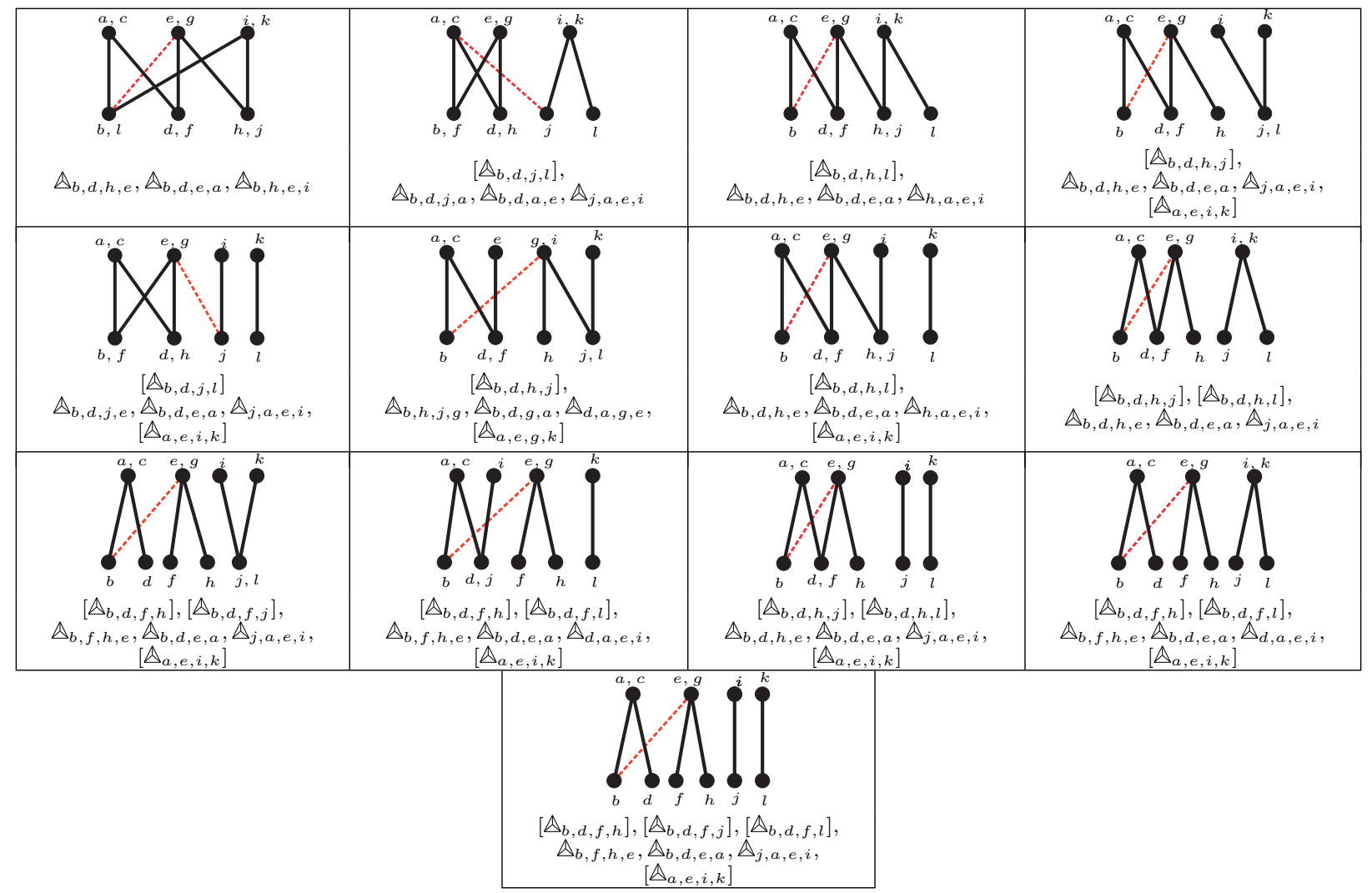

TABLE IV

All POSSIBLE TOPOLOGIES FOR A STEWART-Gough PLATFORM THAT REQUIRES TWO VIRTUAL LEGS, E.G. THE ONES SHOWN IN DASHED LINES, TO BECOME TRILATERABLE.

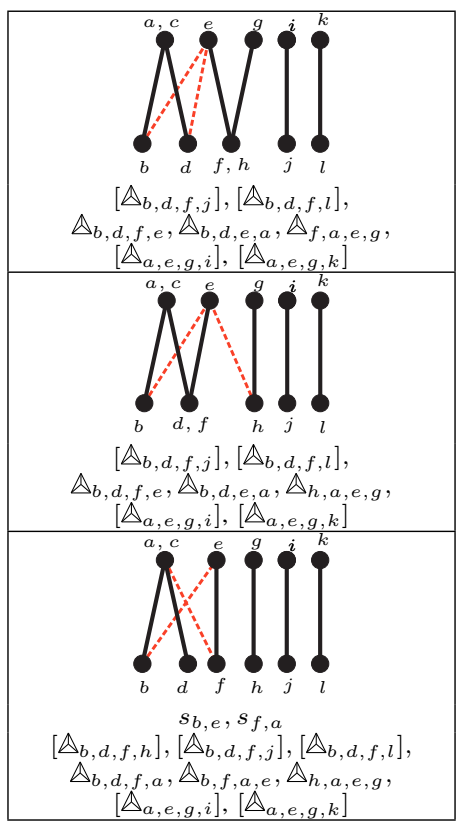

a constant factor, which is dropped here to simplify the formulation. The Cayley-Menger determinants play a central role in distance geometry. For more details on them and their properties see [15], [16].

\section{A. Trilateration in matrix form}

Given $\mathbb{\Delta}_{i, j, k, l}$, the output vector $\mathbf{p}_{i, l}$ can be expressed as a function of the base vectors $\mathbf{p}_{i, j}$ and $\mathbf{p}_{i, k}$ and its squared edge distances as follows (see [8] for details):

$$
\mathbf{p}_{i, l}=\frac{B_{i, j, k, l}}{A_{i, j, k}} \mathbf{p}_{i, j}+\frac{C_{i, j, k, l}}{A_{i, j, k}} \mathbf{p}_{i, k}+\sigma_{i, j, k, l} \frac{D_{i, j, k, l}}{A_{i, j, k}} \mathbf{p}_{i, j, k}
$$

where

$$
\begin{aligned}
A_{i, j, k} & =-D(i, j, k), \\
B_{i, j, k, l} & =D(i, k, l ; i, j, k), \\
C_{i, j, k, l} & =-D(i, j, l ; i, j, k), \\
D_{i, j, k, l} & =\sigma_{i, j, k, l} \sqrt{2 D(i, j, k, l)},
\end{aligned}
$$

and where $\sigma_{i, j, k, l}$ accounts for the two possible locations of $l$ with respect to the plane supporting $\triangle_{i, j, k}$ such that $\sigma_{i, j, k, l}$ is the sign of $\left|\mathbf{p}_{i, j} \mathbf{p}_{i, k} \mathbf{p}_{i, l}\right|$.

Equation (5) can be expressed in matrix form as

$$
\mathbf{p}_{i, l}=\mathbf{Q}_{i, j, k} \boldsymbol{\omega}_{i, j, k, l},
$$


where

$$
\boldsymbol{\omega}_{i, j, k, l}=\left(\frac{B_{i, j, k, l}}{A_{i, j, k}}, \frac{C_{i, j, k, l}}{A_{i, j, k}}, \frac{D_{i, j, k, l}}{A_{i, j, k}}\right)^{T} .
$$

Using $\mathbf{p}_{i, l}$, we can obtain the vector between $l$ and any other point already expressed in the same base, say $m$, as

$$
\begin{aligned}
\mathbf{p}_{l, m} & =\mathbf{p}_{i, l}-\mathbf{p}_{i, m} \\
& =\mathbf{Q}_{i, j, k} \boldsymbol{\omega}_{i, j, k, l}-\mathbf{Q}_{i, j, k} \boldsymbol{\omega}_{i, j, k, m} \\
& =\mathbf{Q}_{i, j, k} \boldsymbol{\omega}_{l, m}
\end{aligned}
$$

with $\boldsymbol{\omega}_{l, m}=\boldsymbol{\omega}_{i, j, k, l}-\boldsymbol{\omega}_{i, j, k, m}$. Then, the squared distances between $l$ and $m$ is

$$
s_{l, m}=\mathbf{p}_{l, m}^{T} \mathbf{p}_{l, m}=\boldsymbol{\omega}_{l, m}^{T} \mathbf{Q}_{i, j, k}^{T} \mathbf{Q}_{i, j, k} \boldsymbol{\omega}_{l, m} .
$$

These new distances can be used in subsequent trilateration steps.

Observe that all terms in a trilateration depend on the coordinates of the base points, the squared distances between points, and the orientations of the tetrahedrons, if provided. This operation is defined irrespectively of whether or not the involved terms are fixed. If so, the computed coordinates and distances will be constant and, otherwise, they will be symbolic and expressed in terms of the squared length of the virtual leg. In trilaterable mechanisms, all trilaterations rely on constant terms and, thus, a trilateration sequence provides coordinates for all the points in the problem. In problems where a virtual leg has to be introduced, the last trilateration step provides a closure condition: if any of the real legs is not used in the trilateration process, its squared constant length can also be expressed symbolically using (7). Clearly, the two expressions must be equal. Such closure condition can be processed as described next to obtain a minimum degree univariate polynomial in the squared length of the virtual leg.

\section{B. Removing radicals}

The closure condition derived using the procedure described in the previous section includes radical expressions. As trilateration steps are applied in sequence, radicals will appear nested and clearing them will consist in an iterative process starting from the outer one. At each step of this process, the expressions involving a radical will have the general form

$$
\alpha_{0}+\alpha_{1} \sqrt{r}+\alpha_{2}(\sqrt{r})^{2}+\alpha_{3}(\sqrt{r})^{3}+\cdots=0,
$$

where $r$ and $\alpha_{i}$ are symbolic expressions. This equation can be rewritten as

$\left(\alpha_{0}+\alpha_{2} r+\alpha_{4} r^{2}+\ldots\right)+\sqrt{r}\left(\alpha_{1}+\alpha_{3} r+\alpha_{5} r^{2}+\ldots\right)=0$,

which can be unfolded into two equations, one for each sign of $\sqrt{r}$. Since we are interested in the roots of both equations, we obtain their product, which can be written as

$\left(\alpha_{0}+\alpha_{2} r+\alpha_{4} r^{2}+\ldots\right)^{2}-r\left(\alpha_{1}+\alpha_{3} r+\alpha_{5} r^{2}+\ldots\right)^{2}=0$,

which does not include the radical any more. By repeating this procedure, all radicals can be cleared.

\section{Eliminating singularity factors}

While clearing radicals as explained above introduces no extraneous roots, one cannot expect to obtain the minimal degree polynomial due to the presence of singularities in the formulation. Indeed, each trilateration step without a fixed base introduces a dividing term, $A_{i, j, k}$, which vanishes when $i, j$, and $k$ are aligned. If this happens, the mechanism can be divided in two parts which can freely rotate about the axis defined by points $i$ and $j$. These degenerated cases have to be considered separately from the general analysis. Assuming that the mechanism does not degenerate, i.e., that $A_{i, j, k} \neq 0$, these factors can be removed from the final expression. This can be simply performed by iteratively dividing the expression obtained after clearing radicals by them until the remainder is not null. This finally leads to the sought-after minimum degree univariate closure polynomial.

\section{Obtaining coordinates}

Each real root of the obtained polynomial can be used to compute possible coordinates for the points in the problem, as if the mechanisms were trilaterable, following the same trilateration sequence used to derive the closure condition.

Observe that, if the orientation of a trilateration step is not fixed, two mirror locations are obtained for the output point with respect to the trilateration base. The first of such trilaterations will produce 2 sets of possible coordinates, the second 4 , the third 8 , etc. Therefore, we will obtain $2^{N}$ sets of possible coordinates, either real or imaginary, $N$ being the number of trilateration steps without fixed orientation. Only the real solutions yield feasible configurations, compatible with the fixed distances in the mechanism. Typically, this process produces only one real solution, except in the cases where different platform poses define the same lengths for the real and virtual legs, e.g., a robot with a planar base where the solutions are split in two sets symmetric with respect to the base.

\section{NUMERICAL EXAMPLES}

To show the generality of the procedure introduced in this paper, we use a Maple implementation to validate it using two platforms with the topologies of the representatives given in (2) and (3). Both of them are 6-4 platforms which have been studied before using different methodologies. In contrast, here we apply the same procedure for both of them.

It is well-known that the forward kinematics of the first example has a maximum of 16 solutions [17]. Actually, the robot described in [17] has 16 real solutions, but its base and platform are coplanar. Thus, the solutions are split in two subsets symmetric with respect to the base. To show the ability of the proposed procedure to deal with oriented tetrahedra, we use the same set of parameters but modifying the $z$-coordinates of the points to avoid coplanarity. Thus, in our case, the coordinates of the points of the base in local reference are

$$
\begin{array}{ll}
\mathbf{p}_{b}=(0,0,5)^{\top} & \mathbf{p}_{d}=(5.1962,-3,-1)^{\top} \\
\mathbf{p}_{f}=(10.3923,0,1)^{\top} & \mathbf{p}_{h}=(5.1962,6,-1)^{\top} \\
\mathbf{p}_{j}=(5.1962,9,1)^{\top} & \mathbf{p}_{l}=(0,6,-1)^{\top}
\end{array}
$$



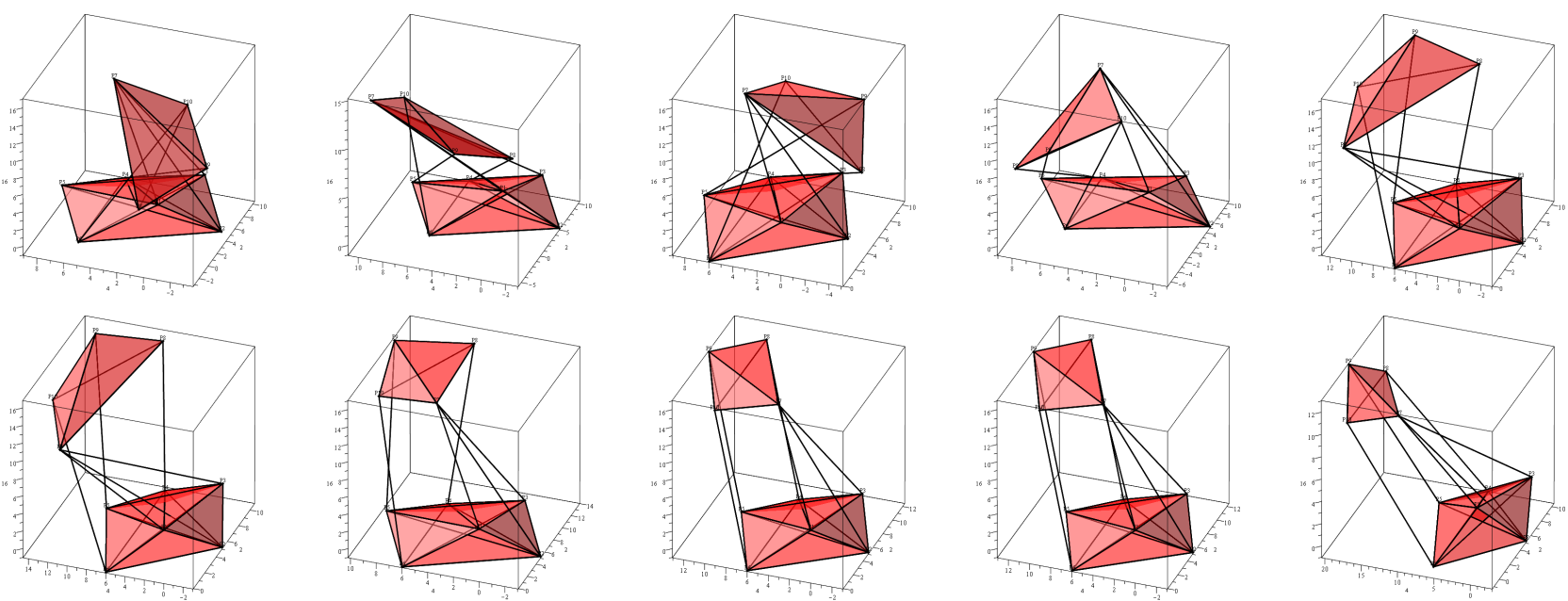

Fig. 3. The 10 different solutions for the forward kinematics of the decoupled platform used to validate the proposed approach.
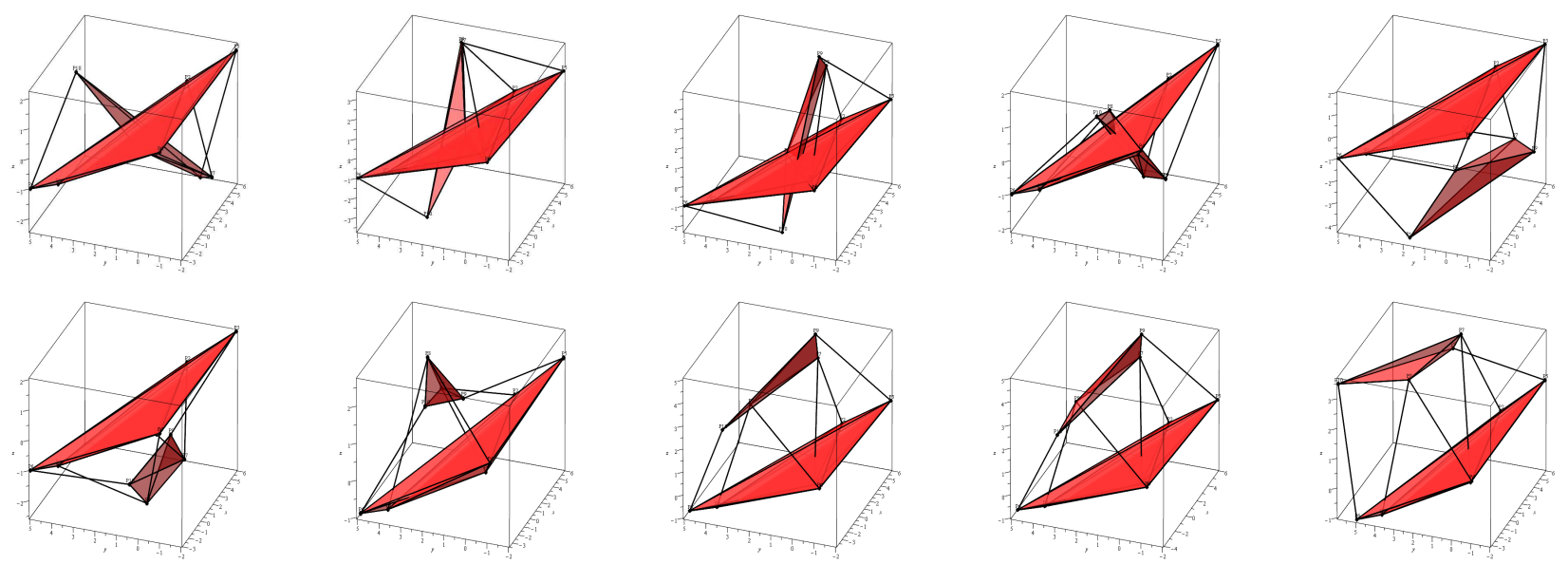

Fig. 4. The 10 different solutions for the forward kinematics of the coupled platform used to validate the proposed approach.

and the coordinates of the attachments in the platform in local reference are

$$
\begin{array}{ll}
\mathbf{p}_{a}=(0,0,1)^{\top} & \mathbf{p}_{e}=(10.3923,6,-1)^{\top} \\
\mathbf{p}_{i}=(5.1962,9,1)^{\top} & \mathbf{p}_{k}=(0,6,-1)^{\top}
\end{array}
$$

The leg lengths are set to

$$
\begin{array}{ll}
d_{a, b}=12.80624847 & d_{a, d}=19.57589575 \\
d_{a, f}=18.50488312 & d_{g, h}=16.61324773 \\
d_{i, j}=16.61324773 & d_{k, l}=16.61324773
\end{array}
$$

After introducing a virtual leg between $b$ and $g$, and using points $b, d$, and $f$ to define a reference, the coordinates of the rest of points and their relative distances can be determined using the trilateration sequence $\left[\triangle_{b, d, f, h}\right],\left[\triangle_{b, d, f, j}\right]$, $\left[\Delta_{b, d, f, l}\right], \Delta_{b, d, f, a}, \Delta_{b, h, a, g}, \Delta_{j, a, g, i}$, and $\left[\triangle_{a, g, i, k}\right]$. Then, the squared constant distance between $k$ and $l, s_{k, l}$, can be also expressed using (7). This provides a closure condition whose solutions are the valid values for $s_{b, g}$. The closure condition includes radical expression, which can be removed using the procedure described in Section III-B. This yields a polynomial in $s_{b, g}$ of degree 40 . Observe, however, that the trilateration steps $\triangle_{b, h, a, g}$ and $\triangle_{j, a, g, i}$ have variable basis which can degenerate. Thus the conditions $A_{b, h, a}=0$ and $A_{j, a, g}=0$ define singularity factors, which have to be removed from the polynomial, as described in Section III-C. After this process we obtain the sought after 16-th degree closure polynomial

$$
\begin{aligned}
& s_{b, g}^{16}-7730.9 s_{b, g}^{15}+2.6910^{7} s_{b, g}^{14}-5.5810^{10} s_{b, g}^{13} \\
& +7.6910^{13} s_{b, g}^{12}-7.4710^{16} s_{b, g}^{11}+5.3110^{19} s_{b, g}^{10} \\
& -2.8110^{22} s_{b, g}^{9}+1.132710^{25} s_{b, g}^{8}-3.4510^{27} s_{b, g}^{7} \\
& +7.9610^{29} s_{b, g}^{6}-1.3610^{32} s_{b, g}^{5}+1.6710^{34} s_{b, g}^{4} \\
& -1.4210^{36} s_{b, g}^{3}+7.8010^{37} s_{b, g}^{2}-2.4310^{39} s_{b, g} \\
& +3.2210^{40} .
\end{aligned}
$$

This polynomial has 10 real solutions which give rise to the 10 configurations shown in Fig 3. The procedure in [12] derives a polynomial of higher degree to account for the two possible orientations of the tetrahedron in the platform.

The second test case is a coupled platform with at most 32 real solutions [14]. The parameters used here are the same 


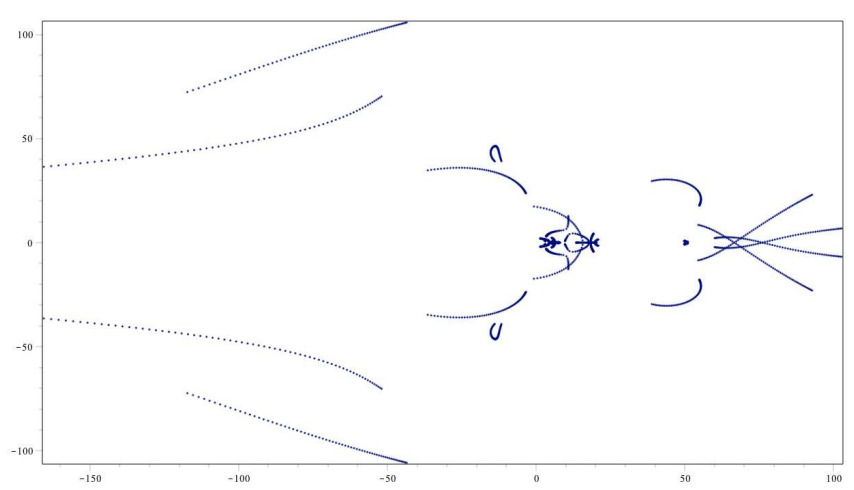

Fig. 5. Solutions of the closure polynomial in the complex plane as we vary the length of the leg providing the closure condition.

as in [18]. Thus, the coordinates of the points of the base and of the platform in local reference are, respectively,

$$
\begin{array}{ll}
\mathbf{p}_{b}=(0,0,0)^{\top} & \mathbf{p}_{d}=(5,0,1)^{\top} \\
\mathbf{p}_{f}=(-2,4,-1)^{\top} & \mathbf{p}_{h}=(3,-1,1)^{\top} \\
\mathbf{p}_{j}=(6,-2,2)^{\top} & \mathbf{p}_{l}=(-3,5,1)^{\top}
\end{array}
$$

and

$$
\begin{array}{ll}
\mathbf{p}_{a}=(4,1,4)^{\top} & \mathbf{p}_{e}=(0,3,3)^{\top} \\
\mathbf{p}_{i}=(4,1,5)^{\top} & \mathbf{p}_{k}=(-4,3,3)^{\top} .
\end{array}
$$

Finally, the leg lengths are set to

$$
\begin{array}{ll}
d_{a, b}=5,74 & d_{a, d}=3,32 \\
d_{e, f}=4,58 & d_{e, h}=5.39 \\
d_{i, j}=4.69 & d_{k, l}=4.58
\end{array}
$$

In this case, a virtual leg between $b$ and $e$ is introduced and points $b, d$, and $f$ are used to define the reference frame for the rest of points. Their coordinates and relative distances are obtained using the trilateration sequence $\left[\Delta_{b, d, f, h}\right],\left[\Delta_{b, d, f, j}\right],\left[\Delta_{b, d, f, l}\right], \Delta_{b, f, h, e}, \Delta_{b, d, e, a}, \Delta_{j, a, e, i}$, and $\left[\triangle_{a, e, i, k}\right]$. Once the coordinates and distances are computed, the squared distance between $k$ and $l$ provides a closure condition which, after removing the radical terms, is a polynomial of degree 232 . This degree reduces to 32 , the minimum possible, after removing the singularity factors introduced by the trilateration steps $\triangle_{b, f, h, e}, \triangle_{b, d, e, a}$, and $\triangle_{j, a, e, i}$.

For the used parameters, the final closure polynomial has 10 real solutions which yield 10 valid configurations. Such configurations are shown in Fig. 4. The obtained coordinates coincide with the ones given in Table 1 of [18].

An additional advantage of the proposed procedure is that the leg lengths directly appear in the closure polynomial. Thus, we can easily modify such lengths and obtain the new set of solutions. For instance, Fig. 5 shows the solutions of the closure polynomial in the complex plane as we vary the length of the leg between $k$ and $l$, i.e, the leg used to generate the closure condition.

\section{CONCLUSION}

This paper introduces a novel perspective on the GoughStewart forward kinematics by elaborating on the idea of using virtual legs. We have shown that there are parallel platform requiring $0,1,2$, or 3 virtual legs to become trivially solvable by trilateration. Since the set of platforms requiring one virtual leg can be divided in two groups, the whole variety of parallel platforms can be classified in five classes. This means that only five representatives need to be considered. Each of them give an idea of the complexity of the corresponding forward kinematics problem. In this paper, we have proved that three of these representatives and, thus, 30 out of 34 possible topologies for a Gough-Stewart platforms, can be solved without variable elimination. This result is remarkable since up to now different solving procedures have been used for particular topologies in these classes that typically rely on variable elimination.

Our current effort focus on extending the presented procedure to solve the representative of the platform topologies requiring two virtual legs and eventually solving the general Gough-Stewart platform forward kinematics.

\section{REFERENCES}

[1] J.-P. Merlet, Parallel Robots. Springer, 2000.

[2] F. Ronga and T. Vust, "Stewart platforms without computer?" in Proc. of the 1992 Int. Conf. in Real Analytic and Algebraic Geometry. Walter de Gruyter, 1995, pp. 196-212.

[3] C. Wampler, "Forward displacement analysis of general six-in-parallel SPS (Stewart) platform manipulators using soma coordinates," Mech. Mach. Theory, vol. 31, no. 3, pp. 331-337, 1996.

[4] M. Husty, "An algorithm for solving the direct kinematics of general Stewart-Gough platforms," Mech. Mach. Theory, vol. 31, no. 4, pp. 365-380, 1996.

[5] P. Dietmaier, The Stewart-Gough platform of general geometry can have 40 real postures. Kluwer Academic Publishers, 1998, pp. 7-16.

[6] I. A. Bonev and J. Ryu, "A new method for solving the direct kinematics of general 6-6 Stewart platforms using three linear extra sensors," Mech. Mach. Theory, vol. 35, no. 3, pp. 423-436, 2000.

[7] N. Trawny, X. S. Zhou, K. X. Zhou, and S. I. Roumeliotis, "3D relative pose estimation from distance-only measurements," IEEE/RSJ International Conference on Intelligent Robots and Systems, 2007.

[8] F. Thomas and L. Ros, "Revisiting trilateration for robot localization," IEEE Transactions on Robotics, vol. 21, no. 1, pp. 93-101, 2005.

[9] J. M. Porta, L. Ros, and F. Thomas, "On the trilaterable six-degreeof-freedom parallel and serial manipulators," in IEEE International Conference on Robotics and Automation, 2005, pp. 960-967.

[10] — - "Inverse kinematics by distance matrix completion," in International Workshop on Computational Kinematics, 2005.

[11] M. Alberich-Carramiñana, F. Thomas, and C. Torras, "Flagged parallel manipulators," IEEE Transactions on Robotics, vol. 23, no. 5, pp. 1013-1023, 2007.

[12] J. M. Porta and F. Thomas, "Closed-form position analysis of variable geometry trusses," Mech. Mach. Theory, vol. 109, pp. 14-21, 2017.

[13] N. Rojas and F. Thomas, "Forward kinematics of the general triple-arm robot using a distance-based formulation," in International Workshop on Computational Kinematics, ser. Mechanisms and Machine Science, vol. 50. Springer, 2018, pp. 257-264.

[14] J. Faugère and D. Lazard, "Combinatorial classes of parallel manipulators," Mech. Mach. Theory, vol. 30, no. 6, pp. 765-776, 1995.

[15] T. Havel, "Some examples of the use of distances as coordinates for Euclidean geometry," Journal of Symbolic Computation, vol. 11, no. 5-6, pp. 579-593, 1991.

[16] J. M. Porta, L. Ros, F. Thomas, and C. Torras, "A branch-andprune algorithm for solving systems of distance constraints," in IEEE International Conference on Robotics and Automation, 2003, pp. 342 348.

[17] M. Hussain and K. J. Waldron, "Direct position kinematics of the 3-1-1-1 Stewart platforms," Transactions of the ASME, vol. 116, pp. $1102-1107,1994$

[18] C. Innocenti, "Direct kinematicsin analytical form of the 6-4 fullyparallel mechanism," Journal of Mechanical Design, vol. 117, pp. 8995, 1995. 